\title{
Efficient EUD Parsing
}

\author{
Mathieu Dehouck Mark Anderson Carlos Gómez-Rodríguez \\ Universidade da Coruña, CITIC \\ FASTPARSE Lab, LyS Research Group, \\ Departamento de Ciencias de la Computación y Tecnologías de la Información \\ Campus Elviña, s/n, 15071 A Coruña, Spain \\ \{mathieu.dehouck,m.anderson,carlos.gomez\}@udc.es
}

\begin{abstract}
We present the system submission from the FASTPARSE team for the EUD Shared Task at IWPT 2020. We engaged with the task by focusing on efficiency. For this we considered training costs and inference efficiency. Our models are a combination of distilled neural dependency parsers and a rule-based system that projects UD trees into EUD graphs. We obtained an average ELAS of 74.04 for our official submission, ranking 4th overall.
\end{abstract}

\section{Introduction}

Latterly, the environmental impact of AI and NLP's dependency on deep neural networks has come under scrutiny (Schwartz et al., 2019; Strubell et al., 2019). This has coincided with a renewed push for efficiency in NLP so as to make systems more easily used in different contexts, be it in hardware impaired conditions, large web-scale applications, or a host of other considerations (Strzyz et al., 2019; Clark et al., 2019; Vilares et al., 2019; Junczys-Dowmunt et al., 2018).

Here we describe our contribution to the Enhanced Universal Dependencies (EUD) Shared Task at IWPT 2020 (Bouma et al., 2020), where we have considered efficiency as well as bare accuracy performance. We combine linguistics and machine learning to develop efficient parsers, both with respect to training and inference. First we curtail the amount of training data we use, second we try distillation to create smaller networks for dependency parsers while maintaining accuracy, and third we develop a rule-based system to cast universal dependency (UD) trees as EUD graphs.

\subsection{An aside on enhanced graphs}

Certain syntactic phenomena, such as the propagation of conjuncts or coreferences in relative clauses, can only be handled implicitly by Universal Dependency (UD) trees resulting in opaque relations or long paths between related content words. EUD graphs is an enhanced representation which can handle these phenomena explicitly. As nodes are not restricted to a single head, these more complex relations can be more readily represented. While this results in a potentially much more useful and informative representation, it also makes for a more challenging task than vanilla UD parsing.

\section{Forest felling}

Distillation introduces extra training overheads. To mitigate this and to balance our pursuit of inference efficiency with some semblance of training efficiency and considering recent results using distillation suggest larger treebanks suffer greater (Anderson and Gómez-Rodríguez, 2020), we decided to set a limit to the size of training treebanks.

In order to minimise introducing compounding variables that could affect training efficacy, we renormalise the sampled treebanks to follow the same tree length distribution of the original treebank. Where more than one treebank exists for a given language, we took a sample from each treebank renormalised with respect to that treebank and took a sample size so that the contribution from each treebank would follow the same ratio as the full data for that language.

We evaluated what limit to set by testing on 4 languages spanning 3 language families (Uralic, Afro-Asiatic, and Indo-European). The only family to appear in the shared task training data not covered was Dravidian as the only example from this language, Tamil, has too small a treebank to have been useful for this analysis. We also cover two branches of the Indo-European family. Balto-Slavic is covered by Russian as the treebank is rather large and uses the Cyrillic script. Germanic is covered by Dutch, which we chose 


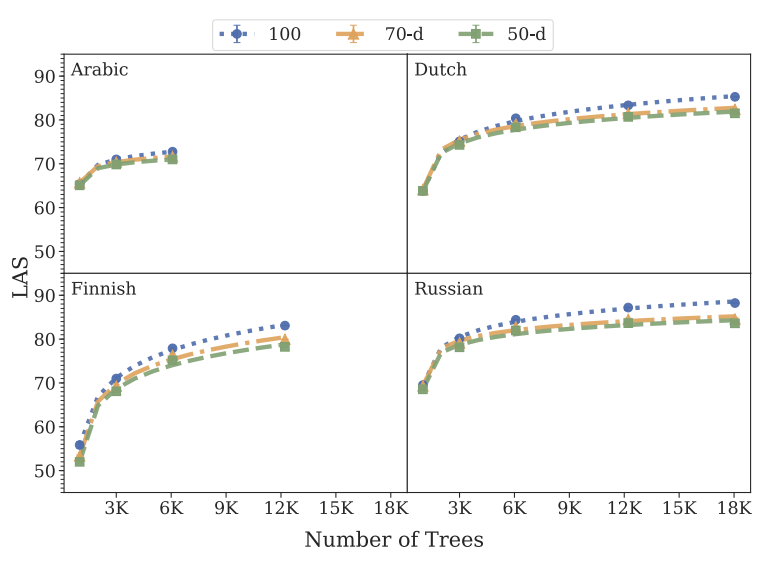

Figure 1: LAS for different models for Arabic, Dutch, Finnish, and Russian development treebanks.

as there are two treebanks which combine to a sizeable number of trees and so would cover the case of combining different treebanks. Finnish was used to cover the Uralic family as we carried this experiment out before the larger Estonian treebank was made available and Arabic was used for Afro-Asiatic. We used sample treebank sizes of $1,000,3,000,6,075$ (the number of trees in the Arabic treebank), 12,217 (the number of trees in the Finnish treebank), and 18,051 (the combined number of trees in both Dutch treebanks). We created 2 splits where possible (i.e. at 6,075 trees Arabic isn't a sample treebank) as a limited attempt at experimental robustness.

We train a Biaffine parser using the hyperparameters of the original paper, shown in Table 6 in the Appendix (Dozat and Manning, 2017). We then distill (as described in Section 3 and in Appendix A.1) these models to two different network sizes, one which has $70 \%$ of the number of nodes in both the BILSTM and MLP layers and one that has $50 \%$. Otherwise the structure of the network is the same as the base model. The LAS averaged over the splits for each sample and model are shown in Figure 1 (similarly for UAS in Figure 8 in Appendix A.1). We are limited by what we can extrapolate from the results for Arabic and Finnish other than they appear to follow a similar trend to Dutch and Russian. For the latter languages we observe the performance levelling at larger treebank sizes, which is neither remarkable nor unexpected, but also a widening between the performance of the full and the distilled models.

As we are concerned with training efficiency, we present the energy consumption for each model type averaged over language and split in Figure 2.

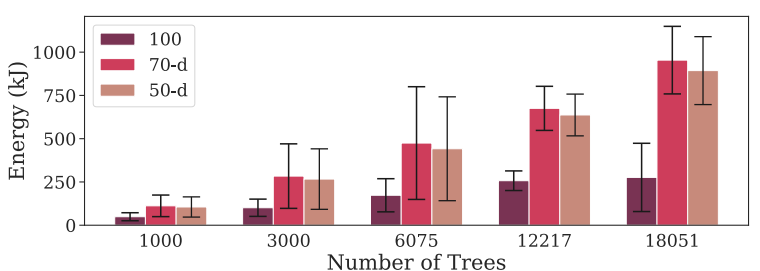

Figure 2: Training energy consumption for different models for different treebank sizes averaged over Arabic, Dutch, Finnish, and Russian.

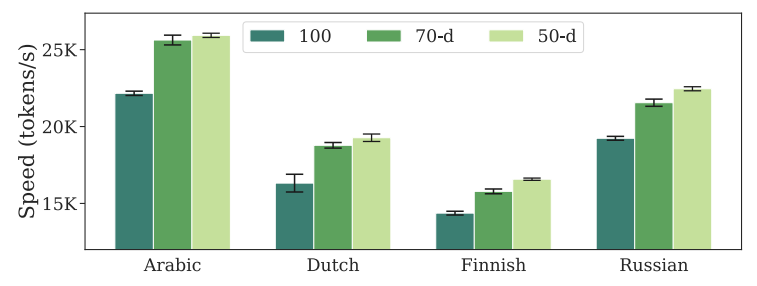

Figure 3: GPU inference speed for different models for treebank 12k (except Arabic which uses its full treebank of 6075 trees) averaged over 5 runs on the development treebanks with batch size 256 .

The amount of energy required to distill our models increases significantly with respect to treebank size. However, distilling to a smaller model requires less energy and, as can be seen in Figure 1, the accuracy difference between the two distilled models is nominal.

Figure 3 shows the inference speed (averaged over splits and 5 runs) on GPU using a single CPU core for each language using the models trained with the 12,217 treebanks (for Arabic we use its full treebank). We observe a sizeable increase in speed over the baseline model for both distilled models, but only a small difference between the two distilled models.

From this, we decided to set an upper limit on the treebank size for the main task to 13,121 (the size of the Italian treebank) as this would require the least amount of tampering and was close to the second largest treebank size used here which performed close to the largest. This meant taking a sample of the Czech, Dutch, Estonian, and Polish treebanks and combining them as described above. A sample was taken for the Russian treebank. Some syntactic metrics are given in Table 7 in the Appendix which shows the different breakdown of the training data used for each of these languages and how they are very similar to the full data. Also, we opted to distill to $50 \%$ of the original model size. For this analysis, and all subsequent analyses, the CPU used was an Intel Core i7- 
7700 and the GPU an Nvidia GeForce GTX $1080 .{ }^{1}$

\section{Boiling neural networks in the pot still}

Neural network compression is not a new phenomenon. For example, pruning has long been shown to be an effective way to reduce parameters with minimal impact on accuracy and also to help generalisation (LeCun et al., 1990; Hagiwara, 1994; Wan et al., 2009; Han et al., 2015; See et al., 2016). However, pruning isn't overly useful for creating efficient models as they leave networks in irregularly sparse states. Other techniques exist that can recast networks into smaller more efficient ones, but we focus on distillation. For a detailed survey of current neural network compression techniques see Cheng et al. (2018).

Anderson and Gómez-Rodríguez (2020) used teacher-student distillation to increase the inference efficiency while only losing marginal accuracy for Universal Dependency (UD) parsing, showing that distilled models outperform models of the same structure and size trained normally. Here we extend that work and use teacher-student distillation to obtain efficient dependency parsers as the basis of our enhanched-dependency parser systems. A full description of our implementation can be found in Anderson and Gómez-Rodríguez (2020) but we also offer a condensed version in Appendix A.1.

While we curtailed our training data, we selected our models based on the performance on the full development data for a given language with gold sentence segmentation and tokenisation. We used characters and words as input to our network. The embeddings for both were randomly initialised. The hyperparameters are the same as used above. We also used early stopping to limit unnecessary training time, stopping after 10 epochs without performance improvement.

At inference time we used UDPipe v2.5 models to predict everything except the parse (Straka and Straková, 2019). When a combination of treebanks were being predicted, we used the model which corresponded to the largest of the treebanks.

Table 1 shows the total time to train the fullsized models and the distillation models for all languages. Also, shown is the GPU energy consumption. The costs for distillation include those of the base models.

\footnotetext{
${ }^{1}$ Using Python 3.7.0, PyTorch 1.0.0, and CUDA 8.0.
}

\begin{tabular}{lcc}
\hline \multicolumn{3}{c}{ Training costs } \\
\hline & Total time & GPU Energy (kJ) \\
Base & 08h:42m:52.1s & 3570.7 \\
Distill & 30h:07m:49.6s & 9981.8 \\
Rule-based & 00h:00m:41.1s & $n / a$ \\
\hline
\end{tabular}

Table 1: Total training time and GPU energy consumption for all treebanks.

Training costs for distillation are more than three times that of the baseline which is hardly surprising. The inference energy cost for all development treebanks (37K trees) for the full model is $2.10(0.09) \mathrm{kJ}$ (average value over 5 runs for each treebank) whereas the cost for distillation is 1.49 $(0.03) \mathrm{kJ}$. Based on these measurements, we would need to parse $390 \mathrm{M}$ sentences to offset the extra cost of distilling models when running on GPU.

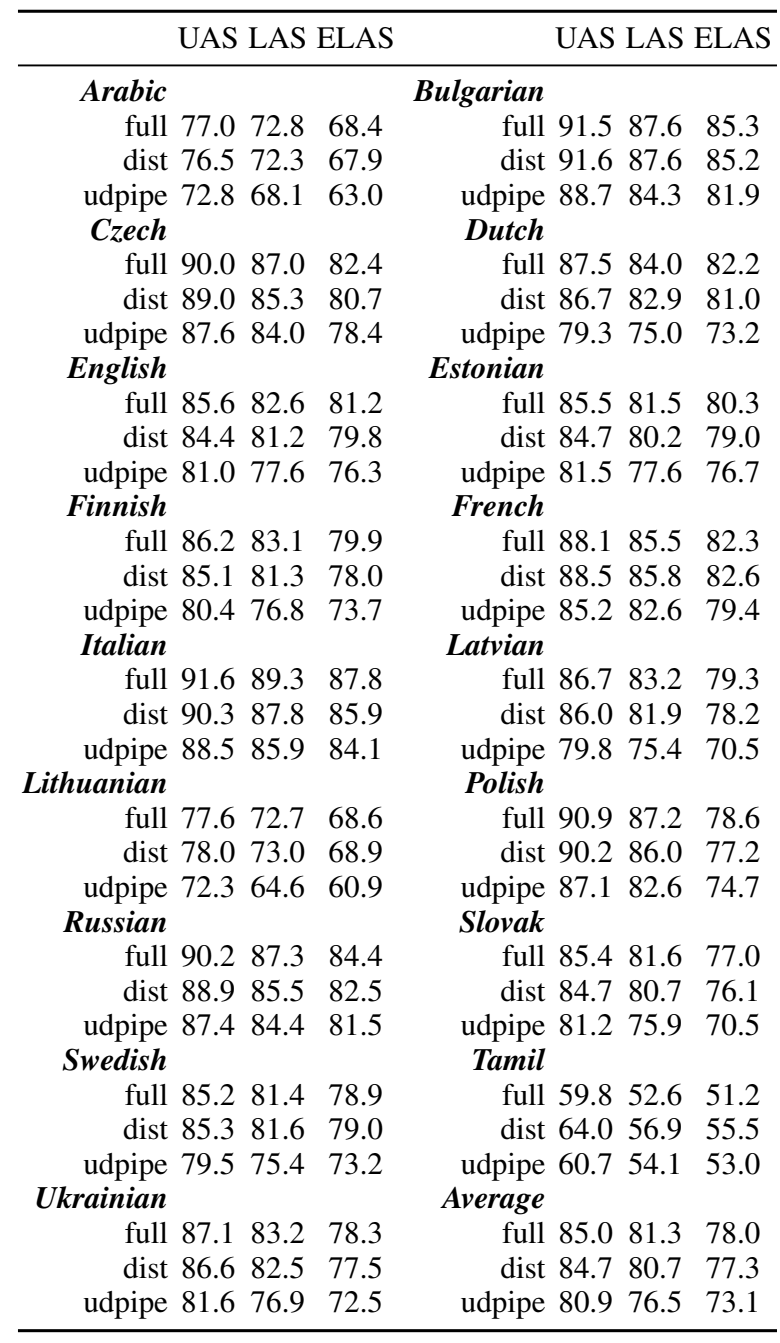

Table 2: Attachment scores for both UD trees and EUD graphs for the development treebanks using different dependency parsers: full baseline models (Full), distilled models (dist), and UDPipe v2.5 models (udpipe). 
Late in the day we decided to validate the results of Anderson and Gómez-Rodríguez (2020), namely that distilled models outperform models trained normally of equivalent sizes. This highlighted that our distilled models used for our official score had not converged. We trained new distilled models and the results given here are for these new models. Our official results using the partially-trained models are in table 9 in the Appendix. All results, including training costs, in this section are for the full-trained distilled models and unless otherwise stated are using the combined development treebanks for each language.

Table 8 in the Appendix shows the performance for the equivalent-sized models trained normally (small) and the distilled models (dist) with respect to UAS and LAS. For the most part the normal models outperform the distilled models. The main differences between our work and that of Anderson and Gómez-Rodríguez (2020) is we do not use pre-trained word embeddings nor POS tags as features. So perhaps without this extra information distillation is less effective. Also, dropout wasn't used during distillation in the original paper but is here, so perhaps the values used here were too punitive a regularisation. Although we use the same hyperparameters as the original paper, the average LAS for the small normally trained models is 0.4 points less than the large model.

We also evaluated the distilled models against the full baseline model and UDPipe v2.5. These results are shown in Table 2. The distilled models outperform the UDPipe models and are within a point of both UAS and LAS to the full model. The ELAS results for the rule-based system using the predicted dependency trees from each of theses systems are also shown. The performance on ELAS generally follows the dependency scores.

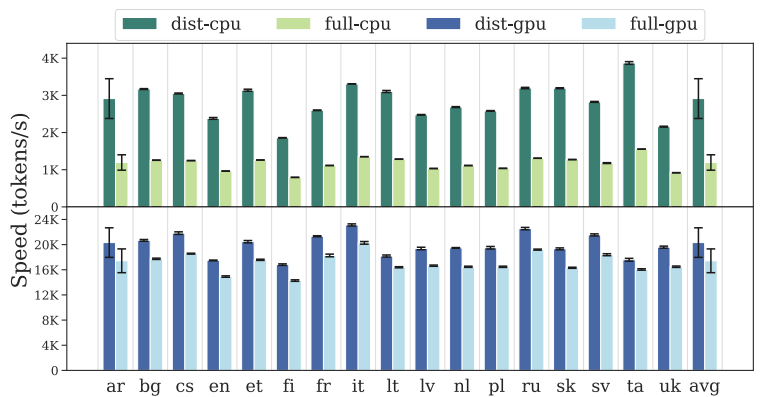

Figure 4: Inference speed for distilled (dist) and full baseline models on CPU (-cpu) and GPU (-gpu) for each development treebank averaged over 5 runs using one CPU core with batch size 256.

\begin{tabular}{llcc}
\hline \multicolumn{3}{c}{ Inference speed (token/s) } \\
\hline \multirow{2}{*}{ CPU } & & UD parser & Full pipeline \\
& Base & $1194.1(207.1)$ & $879.0(123.4)$ \\
& Distill & $2912.9(535.1)$ & $1569.9(238.8)$ \\
& UDPipe & $3629.4(584.0)$ & $2220.2(698.0)$ \\
GPU & & & \\
& Base & $17427.0(1890.3)$ & $2993.3(680.2)$ \\
& Distill & $20321.6(2348.9)$ & $3073.7(714.9)$ \\
\hline
\end{tabular}

Table 3: Inference speeds for dependency parsers and the full EUD pipeline for different systems run on development treebanks and averaged over 5 runs.

Figure 4 shows the inference speed using GPU and CPU of the full baseline model and the distilled models for each language. These are obtained by running the parser 5 times for each language on the full development data and only using one CPU core. The average speed (token/second) increase was $2.44 \mathrm{x}(1.17 \mathrm{x})$ on CPU (GPU).

Table 3 shows the inference speeds for the full pipeline and the dependency parser. We also compare UDPipe inference performance as it is a viable candidate for an efficient parser. It is the fastest of the systems compared here, but the full pipeline which used it obtained an average ELAS 4.9 points less than full baseline model whereas the distilled models are only 0.7 points less.

\section{Unravelling trees with shrewd rules}

Rule-based systems are intrinsically efficient with respect to training time (barely a flash in the pan) and inference time (there is practically none). So we developed a simple rule-based system to enhance the existing dependency tree and reveal hidden dependencies in a cross-lingual setting using as few language specific rules as possible. Beyond the basic enhancement of the original dependencies, there are four main phenomena that create new dependencies: relative clauses, control, conjunction and ellipsis. Since our pipeline does not predict empty nodes, we decided to ignore ellipsis in this system. To deal with each of these phenomena, our algorithm needs to make a number of passes over each sentence.

Pass one - relative clauses and controls: The first pass of the algorithm iterates through each word in the sentence and creates enhanced relations according to the type of the original dependency. When necessary, it adds lemma and case information. If the current word is a relative pronoun/adverb, its antecedent is found by following 
its path to the root until an acl:relcl relation is met. Then a ref edge is created between the word and its antecedent and an edge between the antecedent and the governor of the relativiser with the same relation type as the original relation (if the relative pronoun is the object of a verb then the antecedent becomes the object of that verb). If the word is the dependent of an xcomp relation, the algorithm looks for a subject amongst its controlling predicate's arguments. If a subject is found, it creates an edge between the subject and the current word of type nsubj (:xsubj) (or csub $j$ in the case of a clausal argument). If no subject is available, the current word is stored in a separate list for later processing. If the word is the dependent of a conj relation, it too is stored in a separate list along with all other conjuncts. Whenever we encounter an argument of the type subject, object or oblique, this information is kept for resolving subjects of controlled predicates.

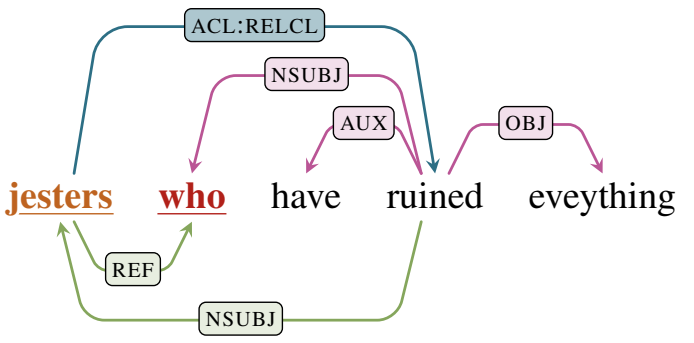

Figure 5: Relative clause example. Pre-existing edges in graph are in magenta and blue. The algorithm observes an acl:relcl relation (highlighted in blue) which causes it to generate two new relations (highlighted in green). A ref relation is created between who and its antecedent, jesters. Then a nsub $j$ is propagated from the head of who, ruined, to jesters.

Pass two - resolving conjunctions: We have two general functions, one for dependent level conjunctions and one for governor level conjunctions, and a few special cases. The dependent level function propagates the conjunction head's original relation to its conjuncts adapting it if necessary, for example in coordinated nmod with different adposition or case. The governor level function propagates the conjunction head's dependents to its conjuncts in the absence of similar dependents and according to morphological agreement. We have a special function that handles subjects of conjuncts because subjects are more diverse than other syntactic functions. In UD at least three relations can mark subjects, namely nsubj for nominal subjects, csub $j$ for clausal subjects and expl used amongst other for syntactic subjects in non prodrop languages (e.g. "it rains"). Subject edges also embed information about their governor, notably information about the voice as : pass when relevant. And, subjects can be absent altogether in prodrop languages, so we rely on morphological information to decide to propagate a given subject in these languages.

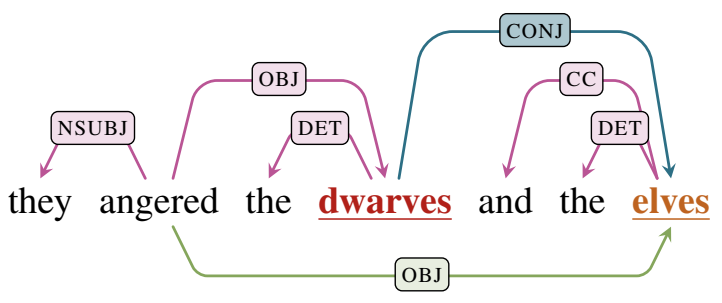

Figure 6: Conjunction example. Magenta and blue edges are those existing in the graph after one pass. During the first pass elves is stored as it is the dependent of a conj relation (highlighted in blue). On the second pass the obj relation of dwarves, the head of this con $j$ relation, propagates to elves generating a new obj relation (highlighted in green) from angered.

Pass three and onwards - sweeping up controls: Once conjunctions have been resolved and more predicates have their arguments stored, the algorithm iterates over controlled predicates that do not have a subject after the first sentence traversal. Several such iterations may be necessary since the number of times a predicate may be coordinated with a controlled verb itself already coordinated to another controlled verb is not bounded. Like in the sentence "Sam stood up and wanted to scream and start running." But in practice one iteration solves the vast majority of missing subjects.

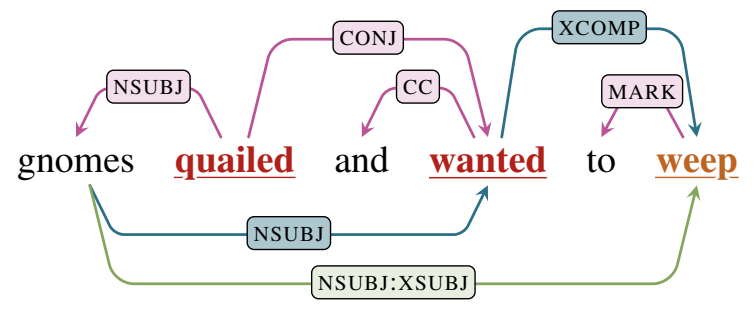

Figure 7: Control example. The edges of the graph after two passes are in magenta and blue. During the first pass weep is stored as it is a dependent of a xcomp relation (highlighted in blue) but it cannot be resolved until wanted is. wanted is resolved in the second pass and an nsubj relation (shown in blue) is propagated from the head, gnomes, of its conjunct, quailed. In the third pass this is further propagated to weep generating a nsub $j$ : xsubj relation (highlighted in green). 


\subsection{Tuning the rules}

A number of enhancements are relation and language specific and some even lexically conditioned such as control, and not all languages include every enhancement type. So the training data is used to tune rules to a given language while keeping the rule definitions as generic as possible.

The first type of information needed regards additional lemmas and cases appearing in edges. For each relation type, the frequency at which case is being added to the relation is obtained. Similarly for lemma, the algorithm counts the frequency of relation types between a word and its dependent used for lexicalisation since different relations are augmented with different dependents (obl usually uses case where acl prefers mark). Furthermore, for lemmas, when several dependents have the same relation, it checks which is used for lexicalisation. For con $j$ though, it only checks if there is anything at all since con $j$ is tightly linked to $\mathrm{CC}$.

Each language is tested to see if it is prodrop by comparing the number of root verbs with an overt subject to the number of root verbs without an overt subject. Whether : xsubj and :relsubj should be added to subjects of controlled predicates and relative clauses is also checked.

The algorithm then checks whether each relation propagates to its governor's conjuncts and under which conditions (the conditions are detailed in Appendix A.2.1) and also if it propagates to its own conjuncts. This is mostly relevant since root usually does not propagate to conjuncts of the main predicate, but in some treebanks it does.

Morphological features are used for detecting relativisers. For each morphological feature, the number of times it co-occurs with a ref enhanced relation is compared to the number of times it co-occurs with another relation. While not an arbitrary choice, it is one of the few cases where an enhanced relation does not depend directly on information in the original tree but on information external to the tree, so in theory we could have chosen other clues such as the lemma of the word instead. These pronouns and adverbs are usually marked with PronType=Rel or PronType=Int, Rel.

Finally, the controlling profile of controlling predicates is learnt. The system discerns which of the arguments is used as subject of controlled verbs and in which conditions, meaning that we do not count subjects in the absence of other arguments since they become default.

\subsection{Problems}

While our rule-based system performs remarkably well, as can be seen in Table 4, with the lowest ELAS being 94.9 on the gold development data, it is challenging to improve across languages simultaneously. Besides the expected ambiguity of language, there are several issues which limit us, some easy to fix, some more complicated, some language specific, and some more general.

\begin{tabular}{lcccc}
\hline \multicolumn{5}{c}{ ELAS } \\
\hline Grabic & Gold & Full & Dist & UDPipe \\
Bulgarian & 98.6 & 68.4 & 67.9 & 63.0 \\
Czech & 97.9 & 82.4 & 85.2 & 81.9 \\
Dutch & 98.9 & 82.2 & 81.0 & 78.4 \\
English & 99.5 & 81.2 & 79.8 & 76.3 \\
Estonian & 99.2 & 80.3 & 79.0 & 76.7 \\
Finnish & 97.3 & 79.9 & 78.0 & 73.7 \\
French & 98.9 & 82.3 & 82.6 & 79.4 \\
Italian & 99.5 & 87.8 & 85.9 & 84.1 \\
Latvian & 95.7 & 79.3 & 78.2 & 70.5 \\
Lithuanian & 98.8 & 68.6 & 68.9 & 60.9 \\
Polish & 94.9 & 78.6 & 77.2 & 74.7 \\
Russian & 98.6 & 84.4 & 82.5 & 81.5 \\
Slovak & 98.8 & 77.0 & 76.1 & 70.5 \\
Swedish & 98.8 & 78.9 & 79.0 & 73.2 \\
Tamil & 99.3 & 51.2 & 55.5 & 53.0 \\
Ukrainian & 95.8 & 78.3 & 77.5 & 72.5 \\
Average & 98.2 & 78.0 & 77.3 & 73.1 \\
\hline & & & &
\end{tabular}

Table 4: Enhanced labelled attachment score for EUD graphs when using gold labelled dependency development treebanks (gold), predicted treebanks with full baseline models (Full), distilled models (Dist), and using UDPipe v2.5 models (UDPipe).

On the monolingual front, incomplete, erroneous and inconsistent annotations are the biggest problems. Incomplete annotation can occur both at the enhanced dependency and the lower level of annotation. For example in Dutch Alpino, we miss 515 ref relations and thus at least as many enhanced relations from their antecedents, representing two thirds of the missing dependencies. The bulk of these missed references are relative/interrogative pronouns/adverbs that are not annotated with an empty feature column. We wanted to avoid too many language specific rules and ignored them, leading to more than a thousand missing edges. Likewise, in some languages not all relativisers (typically interrogative adverbs) are marked as references when they should be according to UD guidelines. 


\begin{tabular}{lrcccccccccccccc}
\hline & \multicolumn{1}{c}{ Tokens } & \multicolumn{1}{c}{ Words Sentences UPOS XPOS UFeats } & AllTags Lemmas UAS LAS CLAS MLAS BLEX EULAS ELAS \\
\hline Arabic & 100.0 & 94.6 & 82.1 & 88.5 & 84.0 & 84.2 & 82.0 & 88.5 & 76.5 & 72.0 & 68.0 & 57.0 & 63.0 & 70.2 & 67.8 \\
Bulgarian & 99.9 & 99.9 & 94.2 & 97.6 & 94.3 & 95.4 & 93.8 & 94.6 & 92.1 & 88.5 & 84.5 & 78.0 & 77.5 & 87.3 & 86.4 \\
Czech & 99.9 & 99.9 & 93.2 & 97.8 & 90.9 & 90.8 & 89.7 & 97.4 & 88.0 & 84.1 & 80.9 & 70.4 & 78.6 & 82.0 & 79.6 \\
Dutch & 99.7 & 99.7 & 69.3 & 92.6 & 89.9 & 92.0 & 89.0 & 94.4 & 84.5 & 80.8 & 73.7 & 63.5 & 68.0 & 79.3 & 78.7 \\
English & 99.2 & 99.2 & 83.8 & 93.6 & 92.8 & 94.1 & 90.7 & 95.4 & 84.8 & 81.7 & 77.7 & 69.0 & 73.8 & 80.8 & 80.1 \\
Estonian & 99.7 & 99.7 & 90.0 & 95.0 & 96.2 & 92.8 & 91.0 & 90.4 & 82.7 & 78.2 & 75.5 & 67.3 & 66.2 & 77.7 & 76.8 \\
Finnish & 99.7 & 99.7 & 88.7 & 94.8 & 54.5 & 93.0 & 51.8 & 87.1 & 86.1 & 82.6 & 80.0 & 72.1 & 67.0 & 80.8 & 79.4 \\
French & 99.7 & 99.2 & 94.3 & 93.5 & 99.2 & 88.8 & 87.3 & 94.9 & 87.8 & 82.2 & 74.8 & 60.5 & 69.1 & 81.6 & 79.5 \\
Italian & 99.9 & 99.8 & 98.8 & 97.2 & 97.0 & 97.1 & 96.2 & 97.4 & 91.4 & 89.1 & 83.8 & 79.2 & 80.3 & 87.6 & 86.9 \\
Latvian & 99.3 & 99.3 & 98.7 & 93.5 & 84.3 & 89.5 & 83.9 & 92.7 & 86.0 & 81.8 & 78.7 & 65.9 & 72.4 & 79.3 & 77.8 \\
Lithuanian & 99.9 & 99.9 & 87.9 & 90.3 & 80.7 & 81.2 & 79.3 & 88.8 & 75.2 & 69.4 & 66.0 & 48.4 & 56.8 & 66.6 & 64.5 \\
Polish & 99.4 & 99.8 & 97.5 & 96.4 & 84.9 & 83.6 & 80.3 & 95.6 & 90.1 & 85.9 & 82.4 & 62.2 & 77.8 & 84.0 & 77.5 \\
Russian & 99.6 & 99.6 & 98.8 & 97.8 & 99.6 & 85.3 & 85.0 & 96.5 & 89.3 & 86.2 & 83.4 & 65.5 & 80.0 & 84.5 & 83.3 \\
Slovak & 100.0 & 100.0 & 85.3 & 92.9 & 77.1 & 80.3 & 76.7 & 86.6 & 85.6 & 81.5 & 78.0 & 56.8 & 64.8 & 79.8 & 76.7 \\
Swedish & 99.2 & 99.2 & 93.5 & 93.3 & 91.0 & 84.9 & 83.2 & 90.0 & 83.4 & 79.3 & 76.0 & 58.6 & 67.0 & 77.9 & 77.0 \\
Tamil & 99.2 & 94.5 & 97.5 & 81.3 & 76.3 & 80.5 & 75.6 & 84.1 & 62.5 & 53.0 & 48.8 & 39.9 & 43.7 & 53.0 & 51.7 \\
Ukrainian & 99.8 & 99.8 & 96.6 & 94.9 & 84.0 & 84.3 & 83.3 & 93.6 & 85.0 & 81.0 & 76.4 & 59.6 & 70.0 & 78.4 & 76.4 \\
Average & 99.7 & 99.1 & 91.2 & 93.6 & 86.9 & 88.1 & 83.5 & 92.2 & 84.2 & 79.8 & 75.8 & 63.2 & 69.2 & 78.3 & 76.5 \\
\hline
\end{tabular}

Table 5: Test results evaluated through the official submission site and using our updated distilled model. Our official submission results can be seen in Table 9 in the Appendix.

Erroneous annotations can be at lower levels of annotation of the dependency tree, thus when applying rules according to these annotations, erroneous edges are created. For example in English (EWT), there is the sentence "Let me know if this is the appropriate steps that you would like to see," in which that which references steps is analysed as the object of like ("you would like the steps to see" vs. "you would like to see the steps") thus the controlling rule for like makes steps the subject of see in place of you. Annotation errors can also happen in the enhanced structure. In Russian, for example, a number of nominal modifiers have diverging case information in the feature column and in the enhanced relation one, often Case $=G e n$ with nmod:acc, so the predicted enhanced relation nmod: gen conflicts with the actual annotation.

Latvian offers an example of inconsistent annotation, nmod is extended with either the adposition's lemma or the word's case but never both and the selection of lemma or case for any given word is seemingly arbitrary. So it is impossible to devise a rule to address this issue.

However, most of these problems are easily rectified with a system such as ours by checking the agreement of case and lemma information in enhanced relations assuming valid annotation of the underlying data.

On the cross-lingual front, the biggest problem is lack of consistency in annotation conventions. Leaving incomplete annotation aside, there are a number of clear divergences. The most striking example is the way subjects of passive verbs and more generally enhanced relations are handled in French Sequoia. These relations receive an extra (:) enh to differentiate them from canonical relations directly taken from the tree, the presence of the column depends mostly on the number of columns in the relation type, if it is a simple relation then a column is used but when it is already a sub-type with a column between the main type and extra information then no column is added. Not only is this unique to this treebank, but it is also redundant since this information can be directly retrieved by looking at the original tree. There are also a number of more subtle inconsistencies. For example, in languages that add lemma information to con $j$ relations, when the coordinating conjunction is a symbol ( $\&$ or $/)$, most languages just ignore them and keep the bare con $j$ relation. However, Swedish uses the special con $j$ : sym relation.

Beyond these issues, there remain genuine linguistic difficulties. A difficulty common to all languages is the scope of conjunctions and whether to propagate dependents amongst conjuncts or not. This is particularly clear with adverbials and obliques that modify verbs. Due to their broad semantic range, adverbials can propagate from conjunction heads to dependent conjuncts even if they already have other adverbials, as long as they do not conflict semantically. Currently in UD, there is no hierarchy amongst dependents of a word, but there could be a form of scope indexing to distinguish a word's direct dependents from dependents of the whole conjunction attached to its head. 
Another difficulty is subject selection in prodrop languages. Fortunately, the prodrop languages in this shared task have personal and number agreement at least on finite verbs which helps testing the compatibility of the overt subject of a verb with its coordinated verbs or verbs in relative clauses that lack an overt subject. However, there are prodrop languages that do not mark personal agreement on verbs and do not use relativisers either (e.g. Japanese). In this case, finding the semantic subject of verbs may be much more challenging.

\section{Results and Discussion}

Despite focusing on efficiency, our official submission obtained an average ELAS of 74.04 which was the fourth best system (out of 9 full submissions). Our improved score after training distilled models to convergence (or closer to convergence) obtained an average score of 76.14. The full breakdown of these results are shown in Table 5 and Table 9 in the Appendix.

Our system is competitive mainly by the grace of our rule-based system which obtains an average 98.20 ELAS when used on the gold development treebanks. And for the most part its performance echoes the quality of the predicted dependencies and tags used by the system as is seen in Table 2. Having a rule-based system that can perform so well on gold data means that improving the dependency predictions it is based on for a full pipeline will almost always increase ELAS scores. It also means it could be used to generate new data. Although this would be restricted to generating data for pre-existing UD treebanks. Furthermore, it could be used to highlight annotation inconsistencies in a given treebank and between different treebanks for the same language.

We also demonstrated that smaller networks can be competitive, even if in this context distillation does not perform as well as previously observed for UD parsing. And beyond that, we show that it is possible to train competitive models with less data and by doing so lowering the energy cost of training parsers. One potentially interesting result is that Tamil performs noticeably better with distillation than either the full baseline model or the small model of the same size trained normally. It has the smallest training treebank out of all the treebanks used in the shared task. The other smaller treebanks also perform better with distillation, e.g the next three smallest treebanks French, Lithuanian, and Swedish all follow this trend but the increase in performance is less pronounced. Perhaps smaller treebanks benefit from what is essentially ensemble training as it tempers a network's penchant for over-fitting.

\section{Acknowledgments}

This work has received funding from the European Research Council (ERC), under the European Union's Horizon 2020 research and innovation programme (FASTPARSE, grant agreement No 714150), from the ANSWER-ASAP project (TIN2017-85160-C2-1-R) from MINECO, and from Xunta de Galicia (ED431B 2017/01, ED431G 2019/01).

\section{References}

Mark Anderson and Carlos Gómez-Rodríguez. 2020. Distilling neural networks for greener and faster dependency parsing. In Proceedings of the 16th International Conference on Parsing Technologies (IWPT 2020) (In press).

Jimmy Ba and Rich Caruana. 2014. Do deep nets really need to be deep? In Advances in Neural Information Processing Systems, pages 2654-2662.

Gosse Bouma, Djamé Seddah, and Daniel Zeman. 2020. Overview of the IWPT 2020 Shared Task on Parsing into Enhanced Universal Dependencies. In Proceedings of the 16th International Conference on Parsing Technologies and the IWPT 2020 Shared Task on Parsing into Enhanced Universal Dependencies, Seattle, US. Association for Computational Linguistics.

Cristian Bucilă, Rich Caruana, and Alexandru Niculescu-Mizil. 2006. Model compression. In Proceedings of the 12th ACM SIGKDD International Conference on Knowledge Discovery and Data Mining, pages 535-541. ACM.

Yu Cheng, Duo Wang, Pan Zhou, and Tao Zhang. 2018. Model compression and acceleration for deep neural networks: The principles, progress, and challenges. IEEE Signal Processing Magazine, 35(1):126-136.

Kevin Clark, Minh-Thang Luong, Urvashi Khandelwal, Christopher D Manning, and Quoc Le. 2019. BAM! Born-again multi-task networks for natural language understanding. In Proceedings of the 57th Annual Meeting of the Association for Computational Linguistics, pages 5931-5937.

Timothy Dozat and Christopher D Manning. 2017. Deep biaffine attention for neural dependency parsing. Proceedings of the 5th International Conference on Learning Representations. 
Masafumi Hagiwara. 1994. A simple and effective method for removal of hidden units and weights. Neurocomputing, 6(2):207-218.

Song Han, Jeff Pool, John Tran, and William Dally. 2015. Learning both weights and connections for efficient neural network. In Advances in Neural Information Processing Systems, pages 1135-1143.

Geoffrey Hinton, Oriol Vinyals, and Jeff Dean. 2015. Distilling the knowledge in a neural network. arXiv preprint arXiv:1503.02531.

Marcin Junczys-Dowmunt, Roman Grundkiewicz, Tomasz Dwojak, Hieu Hoang, Kenneth Heafield, Tom Neckermann, Frank Seide, Ulrich Germann, Alham Fikri Aji, Nikolay Bogoychev, et al. 2018. Marian: Fast neural machine translation in $\mathrm{C}++$. In Proceedings of ACL 2018, System Demonstrations, pages 116-121.

Yoon Kim and Alexander M Rush. 2016. Sequencelevel knowledge distillation. In Proceedings of EMNLP, pages 1317-1327.

Adhiguna Kuncoro, Miguel Ballesteros, Lingpeng Kong, Chris Dyer, and Noah A. Smith. 2016. Distilling an ensemble of greedy dependency parsers into one MST parser. In Proceedings of the 2016 Conference on Empirical Methods in Natural Language Processing, pages 1744-1753, Austin, Texas. Association for Computational Linguistics.

Yann LeCun, John S Denker, and Sara A Solla. 1990. Optimal brain damage. In Advances in neural information processing systems, pages 598-605.

Yijia Liu, Wanxiang Che, Huaipeng Zhao, Bing Qin, and Ting Liu. 2018. Distilling knowledge for search-based structured prediction. In Proceedings of the 56th Annual Meeting of the Association for Computational Linguistics (Volume 1: Long Papers), pages 1393-1402, Melbourne, Australia. Association for Computational Linguistics.

Liang Lu, Michelle Guo, and Steve Renals. 2017. Knowledge distillation for small-footprint highway networks. In 2017 IEEE International Conference on Acoustics, Speech and Signal Processing (ICASSP), pages 4820-4824. IEEE.

Roy Schwartz, Jesse Dodge, Noah A Smith, and Oren Etzioni. 2019. Green AI. arXiv preprint arXiv:1907.10597.

Abigail See, Minh-Thang Luong, and Christopher D Manning. 2016. Compression of neural machine translation models via pruning. In Proceedings of The 20th SIGNLL Conference on Computational Natural Language Learning, pages 291-301.

Milan Straka and Jana Straková. 2019. Universal dependencies 2.5 models for UDPipe (2019-12-06). LINDAT/CLARIAH-CZ digital library at the Institute of Formal and Applied Linguistics (ÚFAL), Faculty of Mathematics and Physics, Charles University.
Emma Strubell, Ananya Ganesh, and Andrew McCallum. 2019. Energy and policy considerations for deep learning in NLP. Proceedings of the 56th Annual Meeting of the Association for Computational Linguistics.

Michalina Strzyz, David Vilares, and Carlos GómezRodríguez. 2019. Viable dependency parsing as sequence labeling. In Proceedings of NAACL-HLT, pages $717-723$.

David Vilares, Mostafa Abdou, and Anders Søgaard. 2019. Better, faster, stronger sequence tagging constituent parsers. In Proceedings of the 2019 Conference of the North American Chapter of the Association for Computational Linguistics: Human Language Technologies, Volume 1 (Long and Short Papers), pages 3372-3383.

Weishui Wan, Shingo Mabu, Kaoru Shimada, Kotaro Hirasawa, and Jinglu Hu. 2009. Enhancing the generalization ability of neural networks through controlling the hidden layers. Applied Soft Computing, 9(1):404-414.

Seunghak Yu, Nilesh Kulkarni, Haejun Lee, and Jihie Kim. 2018. On-device neural language model based word prediction. In Proceedings of the 27th International Conference on Computational Linguistics: System Demonstrations, pages 128-131.

\section{A Appendix}

\section{A.1 Teacher-student distillation}

Model distillation is the act of taking one or more models and guiding the training of a single network with these models. It was originally introduced not as a means of creating more efficient models, but as a way of ensemble training with networks (Bucilă et al., 2006; Ba and Caruana, 2014; Hinton et al., 2015; Kuncoro et al., 2016).

Teacher-student distillation, the method used in this work, has been successfully utilised in a number of NLP tasks ranging from machine translation, language modelling, exploring structured linguistic space, and speech recognition (Kim and Rush, 2016; Lu et al., 2017; Liu et al., 2018; Yu et al., 2018).

In teacher-student distillation, the teacher guides the training of another model, the student, which in our experiments is smaller. The student explicitly uses the information of the larger model by comparing the probability distribution of the respective model's output layer. We use the Kullback-Leibler divergence to obtain the loss between these two distributions:

$$
\mathcal{L}_{K L}=-\sum_{t \in b} \sum_{i} P\left(\mathbf{x}_{i}\right) \log \frac{P\left(\mathbf{x}_{i}\right)}{Q\left(\mathbf{x}_{i}\right)}
$$


where $P$ is the probability distribution from the teacher's softmax layer, $Q$ is the probability distribution from the student's, and $\mathbf{x}_{i}$ is input vector to the softmax corresponding to token $w_{i}$ of a given tree $t$ for all trees in batch $b$.

For our implementation there are two probability distributions as we are using a Biaffine parser, one for head predictions and one for label predictions. $^{2}$

The student is also trained directly on the gold heads and labels using a categorical cross entropy loss, e.g. for the loss on head predictions:

$$
\mathcal{L}_{C E}=-\sum_{t \in b} \sum_{i} \log p\left(h_{i} \mid \mathbf{x}_{i}\right)
$$

where $h_{i}$ is the true head position for token $w_{i}$, corresponding to the softmax layer input vector $\mathbf{x}_{i}$, of tree $t$ in batch $b$.

The total loss is therefore the combination of the Kullback-Leibler loss between the probability distributions of the teacher and the student for both head and label predictions with the cross entropy loss between the student predictions and the gold data:

$$
\begin{gathered}
\mathcal{L}=\mathcal{L}_{K L}\left(T_{h}, S_{h}\right)+\mathcal{L}_{K L}\left(T_{l a b}, S_{l a b}\right) \\
+\mathcal{L}_{C E}(h)+\mathcal{L}_{C E}(l a b)
\end{gathered}
$$

where $\mathcal{L}_{C E}(h)$ is the loss for the student's predicted head positions, $\mathcal{L}_{C E}(l a b)$ is the loss for the student's predicted arc label, $\mathcal{L}_{K L}\left(T_{h}, S_{h}\right)$ is the loss between the teacher's probability distribution for arc predictions and that of the student, and $\mathcal{L}_{K L}\left(T_{l a b}, S_{l a b}\right)$ is the loss between label distributions.

\section{A.2 Details of dependency enhancements}

In this section we give more details about the enhancement of dependency relations and about the processing subtleties of relative clauses, controlled predicates, and conjunctions.

Most of the original dependencies are kept in the enhanced structure, but they can undergo a number of cosmetic changes. In the simplest case, the relation type $t$ is just appended to the index $h$ of the word's governor to give the relation $h: t$. Sometimes, during the process the relation type is slightly modified. In Estonian (EDT and EWT) some complex relations such as compound:prt

\footnotetext{
${ }^{2}$ The PyTorch implementation used can be found at: www.github.com/zysite/biaffine-parser
}

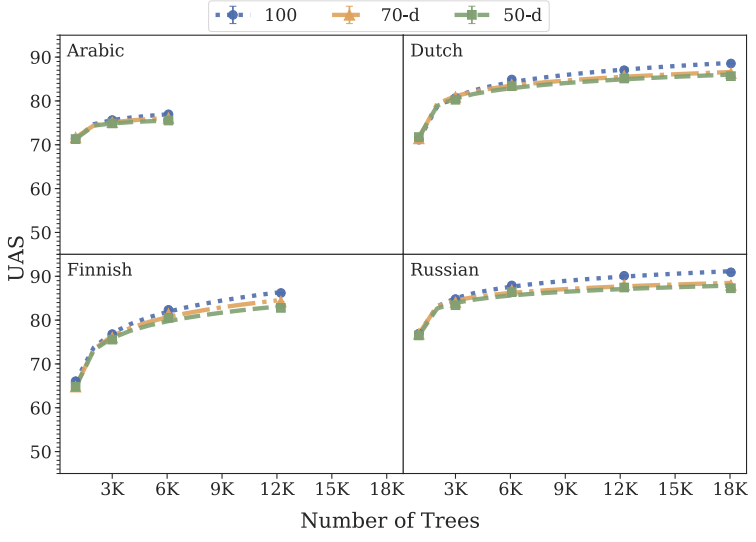

Figure 8: UAS for different models for Arabic, Dutch, Finnish, and Russian development treebanks.

\begin{tabular}{lr}
\hline hyperparameter & value \\
\hline word embedding dimensions & 100 \\
char embedding dimensions & 32 \\
char BiLSTM dimensions & 100 \\
embedding dropout & 0.33 \\
BiLSTM dimensions & $400(200)$ \\
BiLSTM layers & 3 \\
arc MLP dimensions & $500(250)$ \\
label MLP dimensions & $100(50)$ \\
MLP layers & 1 \\
learning rate & 0.2 \\
dropout & 0.33 \\
momentum & 0.9 \\
L2 norm $\lambda$ & 0.9 \\
annealing & $0.75^{\wedge}(t / 5000)$ \\
$\epsilon$ & $1 \times 10^{-12}$ \\
optimiser & Adam \\
loss function & cross entropy \\
epochs & 100 \\
min vocab freq. & 2 \\
\hline
\end{tabular}

Table 6: Hyperparameters for baseline models. The values in parentheses show the values for the distilled and small models used in the main analysis of the shared task.

or csubj:cop are truncated and only the first part is kept. Conversely, in French (Sequoia) some relations receive extra information, such as subjects of passives nsubj:pass that are augmented with xoxobj stating they are the semantic object of their head.

Some relations receive extra lexical and morphological information. Conjuncts marked with conj usually receive the lemma of the coordinating conjunction $(\mathrm{Cc})$. Likewise, adverbial and adjectival clauses (advcl and acl) receive the lemma of the word (mark) that introduces them. Nominal modifiers and obliques (nmod and obl) can receive the lemma of the adposition that introduces them (often marked with the case re- 


\begin{tabular}{|c|c|c|c|c|c|c|c|c|c|}
\hline & \multicolumn{4}{|c|}{ original } & \multicolumn{4}{|c|}{ sample } & \multirow[b]{2}{*}{$2 s-K S_{I}$} \\
\hline & trees & $\mathrm{mL}$ & $\mathrm{mDD}$ & $\mathrm{NP} \%$ & trees & $\mathrm{mL}$ & $\mathrm{mDD}$ & NP\% & \\
\hline \multicolumn{10}{|l|}{ Czech } \\
\hline$-\mathrm{CAC}$ & 23478 & 20.1 & 3.7 & 2.5 & 3016 & 19.9 & 3.7 & 2.5 & 0.014 \\
\hline -FicTree & 10160 & 13.2 & 3.6 & 3.8 & 1305 & 13.1 & 3.6 & 3.8 & 0.016 \\
\hline -PDT & 68495 & 17.1 & 3.7 & 2.7 & 8800 & 17.1 & 3.7 & 2.6 & 0.007 \\
\hline -combined & 102133 & 17.4 & 3.7 & 2.7 & 13121 & 17.4 & 3.7 & 2.7 & 0.007 \\
\hline \multicolumn{10}{|l|}{ Dutch } \\
\hline -Alpino & 12264 & 15.2 & 4.0 & 4.5 & 8915 & 15.2 & 4.0 & 4.4 & 0.004 \\
\hline -LassySmall & 5787 & 13.0 & 3.7 & 2.0 & 4206 & 13.0 & 3.7 & 1.9 & 0.007 \\
\hline -combined & 18051 & 14.5 & 3.9 & 3.8 & 13121 & 14.5 & 3.9 & 3.7 & 0.004 \\
\hline \multicolumn{10}{|l|}{ Estonian } \\
\hline -EDT & 24633 & 14.0 & 3.6 & 0.8 & 12552 & 14.1 & 3.6 & 0.8 & 0.010 \\
\hline -EWT & 1116 & 15.4 & 3.8 & 1.5 & 569 & 15.4 & 3.8 & 1.6 & 0.027 \\
\hline -combined & 25749 & 14.1 & 3.6 & 0.9 & 13121 & 14.1 & 3.6 & 0.8 & 0.009 \\
\hline \multicolumn{10}{|l|}{ Polish } \\
\hline -LFG & 13774 & 7.6 & 2.8 & 0.3 & 5738 & 7.6 & 2.8 & 0.3 & 0.006 \\
\hline -PDB & 17722 & 15.9 & 3.4 & 1.4 & 7383 & 15.9 & 3.4 & 1.5 & 0.008 \\
\hline -combined & 31496 & 12.3 & 3.3 & 1.1 & 13121 & 12.3 & 3.3 & 1.2 & 0.003 \\
\hline \multicolumn{10}{|l|}{ Russian } \\
\hline -SynTagRus & 48814 & 17.8 & 3.6 & 1.6 & 13121 & 17.8 & 3.6 & 1.6 & 0.004 \\
\hline
\end{tabular}

Table 7: Analysis of renormalised treebank samples: 2s-KS is the two-sample Kolmogorov-Smirnov test comparing the sentence-length distributions of the original and the sample treebanks (where values close to 0 suggest samples are not from different distributions, and values approaching 1 suggest otherwise); trees is the number of trees; $\mathrm{mL}$ is the mean sentence length; $\mathrm{mDD}$ the mean dependency distance; and $\mathrm{NP} \%$ is the percentage of non-projective arcs. Where we use the combined sample (or just the sample for Russian-SynTagRus) for training.

\begin{tabular}{|c|c|c|}
\hline & UAS LAS & UAS LA \\
\hline Arabic & & Bulgar \\
\hline & 6.972 .5 & 191.687 .6 \\
\hline & 76.57 & t 91.687 .6 \\
\hline Czech & & Dutch \\
\hline & 39.586 .0 & 1187.283 .3 \\
\hline & 89.085 .3 & ist 86.782 .9 \\
\hline English & & Estonian \\
\hline & $\begin{array}{lll}85.0 & 81.9\end{array}$ & 1185.280 .9 \\
\hline & 84.481 .2 & ist 84.780 .2 \\
\hline Finnish & & French \\
\hline & $85.8 \quad 82.2$ & all $88.1 \quad 85.5$ \\
\hline & 85.181 .3 & ist 88.585 .8 \\
\hline Italian & & Latvian \\
\hline & 91.3 & all $86.3 \quad 82.4$ \\
\hline & 90.387 .8 & dist 86.081. \\
\hline huania & & Polish \\
\hline & 76771 & all $90.5 \quad 86.4$ \\
\hline $\mathrm{d}$ & 78.073 .0 & ist 90.286. \\
\hline Russian & & Slovak \\
\hline & 89.586. & small 85.681 .7 \\
\hline & 88.985 .5 & ist $84.780^{\circ}$ \\
\hline Swedish & & Tamil \\
\hline & 84.580 .8 & small $63.7 \quad 55.7$ \\
\hline dist & 85.381 .6 & dist 64.056 .9 \\
\hline Ukrainian & & Average \\
\hline & $86.8 \quad 82.6$ & small 85.080 .9 \\
\hline dist & 86.682 .5 & dist 84.780 .7 \\
\hline
\end{tabular}

Table 8: Comparison of attachment scores for the development treebanks for distilled (dist) models and models with the same parameters (small) trained normally. lation). Furthermore nmod and obl can also receive case information about the word itself. When the introducing marker is not a word but a fixed expression such as "as well as" then the long lemma composed of the lemmas of each word in the expression (marked by the fixed relation) is used, for example conj: as_well_as.

Relative clauses The only relations from the original tree that are not kept in the enhanced structure are those whose dependent is an anaphoric pronoun or adverb used to introduce a relative clause. Instead, the dependent (pronoun or adverb) is linked to its antecedent by an edge labelled ref. A new edge is then added between the original head of the reference and its antecedent of the same type as the original relation in order to show the argument structure of the clause. Thus, relative clauses are the first phenomenon that creates edges that are not present in the original tree. Their structure is however relatively simple since they can at most create one extra edge and replace one.

There are nonetheless two subtleties with relative clauses. First, in some languages, such as English, relative pronouns are not necessary. In these cases, while there are restrictions on the role the antecedent can fill, we need to infer its actual role from the sentence. Second, there may be sev- 


\begin{tabular}{|c|c|c|c|c|c|c|c|c|c|c|c|c|c|c|}
\hline & oke & & & & & & & & 4 & & & & & 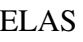 \\
\hline Arabic & 100.0 & 94.6 & 82.1 & 88.5 & 84.0 & 84.2 & 82.0 & 88.5 & 75.871 .2 & 66.8 & 56.1 & 62.0 & 69.2 & 66.9 \\
\hline Bulgarian & 99.9 & 99.9 & 94.2 & 97.6 & 94.3 & 95.4 & 93.8 & 94.6 & 91.187 .0 & 82.3 & 75.8 & 75.5 & 85.8 & 84.9 \\
\hline Czech & 99.9 & 99.9 & 93.2 & 97.8 & 90.9 & 90.8 & 89.7 & 97.4 & 86.281 .8 & 78.1 & 67.9 & 75.8 & 79.6 & 77.2 \\
\hline Dutch & 99.7 & 99.7 & 69.3 & 92.6 & 89.9 & 92.0 & 89.0 & 94.4 & 83.479 .4 & 71.9 & 61.9 & 66.4 & 78.0 & 77.4 \\
\hline English & 99.2 & 99.2 & 83.8 & 93.6 & 92.8 & 94.1 & 90.7 & 95.4 & 83.780 .1 & 75.8 & 67.2 & 72.1 & 79.2 & 78.5 \\
\hline Estonian & 99.7 & 99.7 & 90.0 & 95.0 & 96.2 & 92.8 & 91.0 & 90.4 & 80.775 .5 & 72.7 & 64.6 & 63.8 & 75.0 & 74.1 \\
\hline Finnish & 99.7 & 99.7 & 88.7 & 94.8 & 54.5 & 93.0 & 51.8 & 87.1 & 84.179 .7 & 76.5 & 69.0 & 64.3 & 77.8 & 75.7 \\
\hline French & 99.7 & 99.2 & 94.3 & 93.5 & 99.2 & 88.8 & 87.3 & 94.9 & 87.280 .6 & 72.1 & 58.3 & 66.7 & 80.1 & 77.8 \\
\hline Italian & 99.9 & 99.8 & 98.8 & 97.2 & 97.0 & 97.1 & 96.2 & 97.4 & $90.2 \quad 87.4$ & 81.4 & 76.8 & 77.9 & 85.9 & 84.8 \\
\hline Latvian & 99.3 & 99.3 & 98.7 & 93.5 & 84.3 & 89.5 & 83.9 & 92.7 & 84.479 .7 & 76.1 & 63.6 & 70.0 & 77.2 & 75.6 \\
\hline Lithuanian & 99.9 & 99.9 & 87.9 & 90.3 & 80.7 & 81.2 & 79.3 & 88.8 & 72.966 .3 & 62.6 & 45.9 & 54.3 & 63.7 & 61.4 \\
\hline Polish & 99.4 & 99.8 & 97.5 & 96.4 & 84.9 & 83.6 & 80.3 & 95.6 & 88.483 .4 & 79.4 & 60.1 & 75.0 & 81.4 & 74.5 \\
\hline Russian & 99.6 & 99.6 & 98.8 & 97.8 & 99.6 & 85.3 & 85.0 & 96.5 & $86.8 \quad 83.2$ & 80.0 & 62.8 & 76.7 & 81.7 & 80.3 \\
\hline Slovak & 100.0 & 100.0 & 85.3 & 92.9 & 77.1 & 80.3 & 76.7 & 86.6 & 83.278 .3 & 73.9 & 53.8 & 61.6 & 76.5 & 73.5 \\
\hline Swedish & 99.2 & 99.2 & 93.5 & 93.3 & 91.0 & 84.9 & 83.2 & 90.0 & 82.277 .6 & 73.7 & 56.8 & 64.9 & 76.2 & 75.2 \\
\hline Tamil & 99.2 & 94.5 & 97.5 & 81.3 & 76.3 & 80.5 & 75.6 & 84.1 & 59.648 .8 & 43.6 & 35.5 & 39.6 & 48.1 & 47.0 \\
\hline Ukrainian & 99.8 & 99.8 & 96.6 & 94.9 & 84.0 & 84.3 & 83.3 & 93.6 & 83.478 .7 & 73.6 & 57.8 & 67.4 & 76.2 & 74.0 \\
\hline Average & 99.7 & 99.1 & 91.2 & 93.6 & 86.9 & 88.1 & 83.5 & 92.2 & 82.577 .6 & 73.0 & 60.8 & 66.7 & 76.0 & 74.0 \\
\hline
\end{tabular}

Table 9: Full test results for our official submission using the shared task's submission site for evaluation.

eral words that look like relativisers in a relative clause even outside conjunction. Often, only one of them is a leaf node, the others introducing further embedded clauses. Only in Finnish (TDT) did we find instances of multiple relative pronouns attaching to the same verb and each being marked as the reference of another word in the sentence.

Control A second phenomenon that creates new dependencies is control, where the subject of an embedded clause is not overt and is provided by one of its governor's arguments. For example in the English sentence "I want you to go," the semantic subject of the verb go is the object of the main verb, namely you. In such a case, an additional relation is added to the structure to represent the dependency of the word you to the embedded predicate $g o$. These structures are marked by a xcomp relation between the embedded predicate and its governor in the original tree. The identity of the new subject depends usually on the governing predicate and its argument structure. So it is mostly a matter of knowing the governing profile of each lexical item given their argument structure. For example, the subject of a predicate embedded in a want to clause is the object of the want to clause if present, its subject otherwise. Control is also quite simple since it has a limited span.

Conjunction The vast majority of new edges are created by conjunctions and is much harder to handle than the two previous phenomena. Contrary to relative clauses and control, conjunction has no direction in the sense that it can occur both at the governor level and at the dependent level. In "Mary and Sam bought strawberries," the conjunction "Mary and Sam" occurs at the dependent level and both Mary and Sam are subject of the verb bought. In "Mary bought strawberries and ate them," the conjunction is now at the governor level and Mary is the subject of both bought and ate. So unlike relative clauses where one merely needs to find the relativiser's antecedent higher up in the tree, or control where one needs to look for the controlled subject amongst the arguments of the controlling predicate, conjunctions can have repercussions both higher up and lower down in the structure at the same time.

The easiest case for conjunction is when it occurs at the dependent level. One just needs to propagate the relation existing between the head of the conjunction and its governor to the other conjuncts. In the case of conjunction at the governor level, things are more complicated. While dependents don't tend to propagate up a conjunction chain but only down, they can be blocked by a number of reasons. For example in "Mary bought and ate strawberries," the object strawberries should attach to bought in the tree and only propagate down to ate. But in "Mary spoke and ate strawberries," strawberries should attach to ate and not propagate up to spoke, even though speak can also have direct objects. And in "Mary bought strawberries and ate," strawberries does not propagate down to ate since it appears before it in the sentence. However, the conditions under which certain dependents do or do not propagate 
to their governor's conjuncts are both language and relation specific. In a given language, objects need not behave like subjects nor like determiners or adverbials. Often if a relation slot (object, subject, determiner) is already filled for a given word, it will block the propagation of the same relation from higher up in the conjunction chain, but it need not always be the case, especially with adverbials. But even an empty slot does not always guarantee propagation, especially in case marking and prodrop languages where morphological consideration play a major role as well. So we need to learn the propagation conditions for each relation type on a per language basis.

In our system, we keep track of dependents of con j relations during the first traversal of a sentence and handle them in the second pass. The main reason for not processing conjuncts as soon as they arrive in the sentence is that some of their dependents (objects, adjectives or adverbials) can appear later and thus would require extra processing. For example, in "Mary bought and ate strawberries," the object of both verbs only appears after the conjunct ate, so upon first seeing ate, bought does not have any object to be propagated.

\section{A.2.1 Conjunction propagating conditions}

We use two sets of conditions in order to guide the propagation of dependents to their governor's conjuncts. The first is about relation types already attached to these conjuncts. Usually an object or a subject does not attach to a verb that already has these slots filled. So for each relation, we measure three frequencies. The frequency at which it co-occurs with other types under its main governor (in the tree), the frequency at which it cooccurs with other types under its conjunct governors (in the enhanced structure) and the frequency at which it does not co-occur with other types because it does not propagate to its governor's conjunct. Any relation with which it co-occurs under its main governor cannot be blocking propagation. Then if a relation is more often than not associated with conjunct governors to which the current relation did not propagate, it is considered a blocking relation. In practice this means that a sub $j$ does not propagate to a conjunct of its governor that already has an expl, for example.

The second condition is based on matching morphological information. For every relation and morphological category (tense, case, aspect, and so on), we measure how often the value of a cate- gory agrees or disagrees between the governor and its conjunct (of the same UPOS tag) when the relation propagates and when it does not. If a category disagrees more often than not between conjuncts which the relation did not propagate, then we assume that the category needs to agree for that relation.

\section{A.3 Curious quibbles and questionable jiggery-pokery}

While being above 94.9 ELAS for all languages, our rule-based system could still be improved to better capture enhanced structures. There are three main points for further improvement.

Upon reviewing the code for the rule-based system, we realised that we catch arguments of relative clauses only in presence of a relativiser that receives the ref relation. This means that we miss a number of relations involved in relative clauses. It remained unnoticed because of all the languages in the shared task, most use relative pronouns/adverbs to introduce relative clauses. In fact the only language that does not have relative pronouns, Tamil, is not yet annotated with relative clauses and it might not even be relevant. Our methodology here is to look for an antecedent when we have a relative pronoun, but we could do the opposite and look for potential relative pronouns when we have a relative clause. The latter should indeed be more language agnostic and work even when there are no relativisers involved.

A second point of improvement has to do with subject finding in controlled predicates. In our current system, the controlling behaviour of each controlling construction is gathered from the training data, and if we encounter an out of vocabulary construction at prediction time the subject is used by default. But further consideration showed that the object might be a more sensible default option when available. It would, however, be more interesting to learn the default behaviour on a per language basis.

Thirdly, due to the march of time, we hardcoded a number of heuristic thresholds used to fine-tune the system. For example, to see if a language is prodrop, we compare the number of root verbs with overt subjects with the number of root verbs without a subject. If at least a third of root verbs do not have an overt subject then that language was considered prodrop. This is clearly not satisfying since this ratio can greatly vary from 
language to language and from genre to genre. Furthermore, some languages may not be generally prodrop, but ommit syntactic subjects in impersonal constructions, such as Hebrew, or be prodrop only for certain tenses. 